\title{
Publisher's Note: Precursor of the Laughlin state of hard-core bosons on a two-leg ladder [Phys. Rev. B 96, 014524 (2017)]
}

\author{
Alexandru Petrescu, Marie Piraud, Guillaume Roux, I. P. McCulloch, and Karyn Le Hur
}

Q (Received 14 August 2018; published 22 August 2018)

DOI: 10.1103/PhysRevB.98.059901

This paper was published online on 31 July 2017 with an error in the Acknowledgments. On page 13, the last sentence in the Acknowledgments should read as "K.L.H. acknowledges support from the German DFG Forschergruppe 2414 and Labex PALM." The Acknowledgments have been corrected as of 14 August 2018. The Acknowledgments are incorrect in the printed version of the journal. 